\title{
Nouveau financement hospitalier: transition en douceur ou dans la douleur?
}

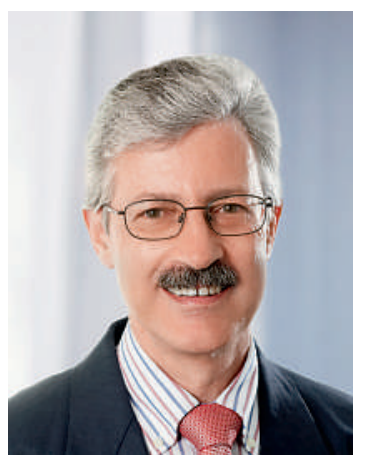

«Des craintes ont été émises de voir la prééminence économique du système prendre le pas sur les soins administrés. A ce point de vue, la direction de l'hôpital tient à exprimer clairement son soutien et sa confiance au corps médical, afin que celui-ci puisse continuer à jouir d'une pleine liberté thérapeutique et traiter tous les patients en fonction de critères d'efficacité prioritaires. S'il est vrai que l'économicité doit être prise en compte, seules des prestations de qualité irréprochable sont à même de garantir la sécurité et la satisfaction des patients.» Cette citation, tirée d'une lettre personnelle adressée il y a quelques jours par la direction à tous les médecins des Hôpitaux valaisans, illustre bien l'aboutissement d'une démarche préparatoire solide et concertée, où médecins, soignants et administrateurs ont pu

\section{«S'il est vrai que l'économicité doit être prise en compte, seules des prestations de qualité irréprochable sont à même de garantir la sécurité et la satisfaction des patients.»}

accorder leur point de vue. Dans un tel cas, les patients n'ont rien à craindre des changements qui auront lieu le $1^{\mathrm{er}}$ janvier prochain, avec l'introduction de la structure tarifaire SwissDRG et la mise en vigueur de tous les autres éléments constitutifs du nouveau financement hospitalier. La plupart des cantons ont procédé à des adaptations législatives et les établissements hospitaliers se sont, pour la majorité d'entre eux, préparés de longue date à cette échéance.

$\mathrm{Au}$ niveau fédéral, la structure tarifaire SwissDRG est prête, dans sa version d'introduction 1.0. Elle représente déjà de manière tout à fait acceptable l'activité effective auprès des patients, la majorité des poids relatifs de coût ayant pu être calculés sur la base des données de nos hôpitaux de référence helvétiques. Dans les modalités d'application, la Confédération a dû intervenir suite au désaccord des partenaires tarifaires: elle a déjà défini la hauteur du calcul des investissements ainsi que le monitorage des prestations. Le serpent de mer de la protection des données devrait prochainement trouver un épilogue, d'une part au Parlement avec le complément proposé par le Conseil fédéral à l'article 42, al. 3 bis et 4 de la LAMal et d'autre part dans l'ordonnance subséquente qui définira le volume et la voie de la transmission des données.

De manière générale, peu de mesures contraignantes ont été décidées au niveau fédéral. En revanche, une grande liberté a été laissée aux cantons et aux partenaires tarifaires, bien dans la ligne de notre tradition fédéraliste. Pour le fi-

\section{Nous devons rester vigilants car il est essentiel d'améliorer chaque année la structure tarifaire et de corriger les éventuels effets néfastes des diverses modifications qui vont survenir.}

nancement de la formation postgraduée des médecins, un consensus a été dégagé, limité à des recommandations étant donné la compétence inaliénable des cantons dans ce domaine. Cependant, bon nombre d'entre eux ont développé des solutions intéressantes, appliquées dès l'an prochain ou au plus tard en 2013. Les négociations du taux de base ont déjà pu aboutir, pour un grand groupe d'assureurs maladie, à un accord satisfaisant avec $90 \%$ des hôpitaux. D'autre part, en cas d'échec des tractations entre partenaires tarifaires, la plupart des cantons imposeront un taux de base provisoire permettant l'établissement des factures et leur encaissement. Il ne devrait ainsi pas y avoir d'insuffisance de financement à court terme dans les soins aigus stationnaires.

Le cadre général est donc propice à une transition en douceur, seules les instances n'ayant pas su anticiper les changements et s'y préparer s'exposant à une période douloureuse. Nous devons cependant rester vigilants car il est essentiel d'améliorer chaque année la structure tarifaire et de corriger les éventuels effets néfastes des diverses modifications qui vont survenir. Pour ce faire, les médecins doivent s'impliquer à tous les niveaux, auprès des directions hospitalières, des autorités cantonales, des partenaires de la santé et des instances fédérales, afin de garantir leur liberté thérapeutique et les intérêts des patients. Dans ce sens, la FMH continuera d'accompagner de manière active et critique les mutations à venir dans le financement hospitalier.

Dr Pierre-François Cuénoud Membre du Comité Central Responsable du Domaine SwissDRG 